\title{
¿ECONOMÍA O SALUD? UN ANÁLISIS GLOBAL DE LA PANDEMIA DE COVID-19"
}

\author{
Liliana Chicaiza Becerra ${ }^{a}$ \\ Mario Garcia Molina \\ Iván Leonardo Urrea
}

" DOI: https://doi.org/10.18601/01245996.v23n44.08. Recepción: 01-092020, modificación final: 12-09-2020, aceptación: 27-11-2020. Sugerencia de citación: Chicaíza-Becerra, L., García-Molina, M. y Urrea I, L. (2021). ¿Economía o salud? Un análisis global de la pandemia de COVID-19. Revista de Economía Institucional, 23(44), 171-194.

a Doctora en Economía y Gestión de la Salud. Profesora titular, Facultad de Ciencias Económicas, Universidad Nacional de Colombia, Bogotá, Colombia, [lachicaizab@unal.edu.co], [https://orcid.org/0000-0001-0002-0003].

b Doctor en Economía. Profesor titular, Facultad de Ciencias Económicas, Universidad Nacional de Colombia, Bogotá, Colombia, [mgarciamo@unal. edu.co], [https://orcid.org/0000-0001-0002-0003].

c Magíster en Ciencias Económicas. Analista de Investigación, Banco Interamericano de Desarrollo y Profesor, Facultad de Ciencias Económicas, Universidad Nacional de Colombia, Bogotá, Colombia, [ilurrear@unal.edu. co], [https://orcid.org/0000-0001-0002-0003]. 


\section{¿Economía o salud? Un análisis global de la pandemia de COVID-19}

Resumen Este artículo estudia la relación entre el manejo sanitario de la pandemia de COVID-19 y los resultados económicos. Identifica algunos indicadores de mortalidad y económicos que toman en cuenta elementos sociales y culturales. Examina el efecto de los precios del petróleo en la capacidad de maniobra de los países exportadores, y el modo en que la disrupción del comercio exterior afectó a las cadenas globales de valor. Los países con mejores resultados en salud durante la pandemia mostraron menores caídas del PIB en el segundo trimentre de 2020. E1 mejor predictor de buenos resultados económicos fue la solidez del sistema de salud en número de camas antes de la pandemia.

Palabras clave: COVID-19, pandemia, crecimiento económico, mortalidad, comercio exterior; JEL: H50, H53, F18

\section{Economy or health? A Global Analysis of the COVID-19 Pandemic}

Abstract This paper identifies the relationship between the health management of the COVID-19 pandemic and the economic outcomes. It shows economic and mortality indicators as well as other social and cultural variables affecting the results. It takes into account the effect of oil prices upon the exporting countries leeway. It also shows how the disruption in international trade affected global value chains. Countries with better health results during the pandemic showed better results in economic growth in the second quarter of 2020. The best predictor of economic success was the health system strengh measured by number of beds prior to the pandemic.

Keywords: COVID-19, pandemic, economic growth, mortality, international trade; JEL: H50, H53, F18

\section{Economia ou saúde? Uma análise global da pandemia COVID-19}

Resumo Este artigo estuda a relação entre a gestão em saúde da pandemia COVID-19 e os resultados econômicos. Identifique alguns indicadores de mortalidade e econômicos que levem em consideração elementos sociais e culturais. Ele examina o efeito dos preços do petróleo na capacidade de manobra dos países exportadores e como a interrupção do comércio exterior afetou as cadeias de valor globais. Os países com os melhores resultados de saúde durante a pandemia mostraram quedas mais baixas do PIB no segundo trimestre de 2020. O melhor indicador de bom desempenho econômico foi a força do sistema de saúde em número de leitos antes da pandemia.

Palavras-chave: COVID-19, pandemia, crescimento econômico, mortalidade, comércio exterior; JEL: H50, H53, F18 
Z1 año 2020 inició con unos cuantos casos reportados de COEVID-19 en Wuhan (China). El contagio se propagó rápidamente por todo el mundo y en menos de seis meses llevó al confinamiento de personas, al cierre de fronteras y cuarentenas estrictas o selectivas que provocaron una profunda crisis económica mundial. Las medidas de confinamiento buscaban contener el número de personas contagiadas que tuvieran que ser tratadas en unidades de cuidado intensivo, cuya disponibilidad depende del nivel de desarrollo del sistema de salud de cada país. Como era de esperar, el confinamiento provocó una reducción de la demanda de productos y de la producción, la quiebra de empresas, despidos de trabajadores y desaceleración de la economía.

Aparentemente, en el manejo de la pandemia se enfrentaba un trade-off entre cuarentenas - para reducir el número de muertes- y el mantenimiento de la economía. Este artículo hace una cronología de las cuarentenas en varios países, presenta cifras de mortalidad por COVID-19, y las contrasta con la contracción del empleo y del crecimiento económico para determinar si los paises eficaces en la reducción de la mortalidad lo hicieron a costa de sacrificar la economía, o no. También se presenta información contextual sobre las variaciones del precio del petróleo y la situación fiscal prepandemia, así como de los efectos de la pandemia en las cadenas de valor del comercio internacional para explorar hipótesis sobre los efectos de algunas variables en el manejo de la crisis.

\section{MARCO METODOLÓGICO PARA EL ANÁLISIS ECONÓMICO}

El análisis de la pandemia y de su impacto económico involucra países muy dispares y efectos de corto plazo para los que no hay suficientes datos comparables. Es apropiado, entonces, utilizar los métodos usuales en historia económica que aprovechan evidencia incompleta para establecer patrones, prestando atención a las diferencias. Solo se incluyeron países que identificaron su primer caso en el primer trimestre de 2020 de los que se obtuvo información confiable, y se identificaron grupos de países medianamente comparables.

Para definir los grupos se tomaron en cuenta varios criterios. El primero distingue entre países que exportan hidrocarburos y países no exportadores, lo cual es relevante porque la pandemia se extendió en un contexto de baja de precios del petróleo, debido al desacuerdo entre Rusia y Arabia Saudita que llevó incluso a precios negativos en algún momento, (Chiaramonti y Maniatis, 2020) y que se reflejaron en el precio internacional de gas natural (Bradley, 2010). Para 
los países en desarrollo que exportan hidrocarburos esto representó una caida de las exportaciones y de los ingresos fiscales que reducía el margen de maniobra en política fiscal. En cambio, los países importadores se vieron beneficiados por los precios. Así, sería de esperar que en los países importadores fuese menor el impacto económico de la pandemia, porque podían subsidiar la nómina e implementar otras políticas de mitigación. Se considera la variable de exportación de hidrocarburos sin dejar de lado otras variables complementarias como el déficit fiscal y el nivel de endeudamiento a finales de 2019.

La segunda variable es el nivel de ingreso según la clasificación del Banco Mundial de 2019. Dada la cronología de la pandemia y la disponibilidad de información, el análisis se concentra en países de ingreso alto y medio.

La tercera variable es el nivel de población, porque en países como India, Brasil, Rusia o Estados Unidos, con una alta población y dispersa en áreas grandes, la propagación de la epidemia es diferente y se comporta como si cada país fuera más bien un conjunto de países. Así, algunas áreas pueden alcanzar el pico de contagios mientras que en otras la infección apenas comienza. Además, varios de esos países, como Estados Unidos y México, tienen un régimen federal y las políticas de cada estado pueden ser muy diferentes. Los países muy grandes tenían más de 200 millones en 2018; los países grandes, entre 100 y 199 millones; medianos, entre 30 y 99 millones; y pequeños, entre 10 y 29 millones de habitantes. No se tomaron en cuenta los países con menos de 10 millones de habitantes (muchos de ellos islas).

Conforme a estas variables se identificaron cinco grupos de países: 1) de ingreso alto, tamaño mediano; 2) de ingreso alto, tamaño pequeño; 3) de ingreso medio alto, tamaño mediano; 4) muy grandes; y 5) exportadores de hidrocarburos. El quinto incluye países con distintos niveles de ingreso. Dentro de de cada grupo se seleccionaron los países con datos disponibles confiables y diferencias apreciables en la aparición del primer caso detectado, para ver en qué medida lo ocurrido en los países de contagio temprano se podía extrapolar, con salvedades, a los países de contagio posterior.

En cada grupo se consideraron variables de resultado final, económico y de salud. Para los resultados económicos se tomaron el mercado laboral y el PIB. Para el mercado laboral, se escogió la tasa de ocupación, pues la variable tradicional -la tasa de desempleo- no es apropiada en esta coyuntura porque las cuarentenas redujeron simultáneamente la población económicamente activa. Mientras que la tasa de ocupación permite apreciar las magnitudes de tamaño 
de la economía, que se pierden en el análisis del número absoluto de empleos perdidos. En países como los latinoamericanos es clave considerar la tasa de informalidad, pues el desempleo disfrazado u ocupación en sectores de baja productividad es importante (Robinson, 1936). Una limitación es que estas cifras no están disponibles para otras regiones en desarrollo, aunque para los países desarrollados se puede hacer abstración del problema. El PIB, la otra variable clara de resultado economico, se tomó en cuenta en la medida de lo posible. No obstante, en muchos países la frecuencia de las observaciones del PIB es más baja que la del empleo. En ambos casos se consideraron los valores a finales de 2019 y el cambio en el segundo trimestre de 2020, además de los datos intermedios cuando estaban disponibles.

En cuanto a las medidas de salud, se escogió la tasa de mortalidad por COVID-19 por millón de habitantes, que es comparable entre países; al menos en mayor medida que las cifras de contagio, que dependen del número de pruebas realizadas. Por ello, en países con políticas basadas en tamizaje de la población y cercos epidemiológicos (como Corea del Sur), las tasas de contagio son mayores que en países con políticas diferentes. Por esa misma razón se excluyó la tasa de letalidad, cuyo denominador es el número de contagiados. Igual que las demás cifras de salud, las de mortalidad son susceptibles de una posible manipulación de los gobiernos, como puede haber sucedido en Nicaragua, y no son utilizables para todos los países. También son propensas a distorsiones, en especial cuando colapsan los servicios de salud, porque el personal médico se dedica al máximo a salvar vidas y no alcanza a registrar las muertes. Esto distorsiona sobre todo las cifras de alta frecuencia; por ejemplo, la cifra de un día puede reflejar datos no contabilizados en la semana anterior, como ocurrió en China y Chile, pero tienden a ser más fiables cuando se examinan promedios mensuales o trimestrales.

La variable ideal para medir los costos en salud sería la mortalidad en exceso por todas las causas, por ejemplo comparando la mortalidad general con la mortalidad general del mismo trimestre del año anterior. Ello captaría un hecho crucial, el problema de la pandemia no es simplemente que la gente muera por el COVID-19 sino que al saturar los servicios de salud, hace más probable que mueran por otras causas aún si no estaban infectados. Esta medida incluso reflejaría el problema de que durante la pandemia y las cuarentenas se posterguen los servicios de salud para casos que no son de urgencias. Esto puede provocar una mayor morbimortalidad en los no contagiados. En el extremo, como sucede con personas con cáncer o leucemias agudas, 
el no diagnosticarlos y tratarlos rápidamente puede conducir a la muerte. Ya están disponibles algunos datos puntuales en este sentido (Mannucci, Nreu y Monami, 2020), pero todavía no hay suficientes para poder hacer comparaciones. Entre tanto, lo segundo mejor son las tasas de mortalidad por COVID-19.

Cuadro 1

Fuentes principales por país

\begin{tabular}{lll}
\hline \multicolumn{1}{c}{ País } & \multicolumn{1}{c}{ Grupo } & \multicolumn{1}{c}{ Fuente } \\
\hline México & Países exportadores & INEGI \\
Colombia & & Federal State Statistics Service \\
Irán & & Dane y Banco de la República \\
Iraq & Central Bank of Iran \\
Bolivia & & Central Bank of Iraq \\
& & Instituto Nacional de Estadística de \\
Ecuador & & Bolivia \\
Argentina & Países de ingreso & Banco Central de Ecuador \\
Malasia & medio alto, tamaño & INDEC \\
Perú & mediano & Instituto Nacional de Estadística e \\
& & Informática \\
Tailandia & & NESDB \\
Australia & Países de ingreso & Australian Government \\
Bélgica & alto, tamaño peque- & National Bank of Belgium \\
Chile & no & Banco Central de Chile \\
Rumania & & Institutul National de Statistica \\
Suecia & & Statistics Sweden \\
Taiwán & & National Statistics, Republic of China \\
Canadá & Países de ingreso & Statistics Canada, Department of \\
& alto, tamaño me- & Finance \\
Alemania & diano & Federal Statistics Office \\
Corea del Sur & & Ministry of Strategy and Finance \\
España & & National Statistics Institute \\
Francia & & INSEE \\
Reino Unido & & Office for National Statistics \\
Italia & & ISTAT \\
Brasil & Países muy grandes & IBGE \\
China & & National Bureau of Statistics of China \\
India & & Centre for Monitoring Indian Eco- \\
& & nomy \\
Estados Unidos & & U.S Bureau of Labor Statistics y U.S. \\
& & Bureau of Economic Analysis \\
\hline
\end{tabular}

Fuente: elaboración propia. 
Las demás variables consideradas fueron las de política o que podían afectar las comparaciones, como el momento del primer contagio y si el país tenía experiencia en manejo de epidemias similares, como en Asia Oriental (Japón, Corea) y Suroriental (Malasia, Tailandia). También hay variables que pueden haber sido cruciales, como la costumbre de usar tapabocas (incluso por razones estéticas como en Japón) y la distancia social aceptable en la vida cotidiana, que varía entre países (Sorokowska et al., 2017; Hall, 1966). Una variable adicional fue qué tan rápida y tan estricta fue la cuarentena.

$\mathrm{E} 1$ análisis por países o grupos de países similares esconde otro factor relevante, el de las relaciones económicas dentro de la globalización. Por ello se complementó considerando lo que pasó con las cadenas internacionales de valor durante la pandemia.

Las fuentes de los datos utilizados por país se indican en el cuadro 1 (el PIB per cápita de paridad de poder adquisitivo corresponde a los cálculos del Banco Mundial para 2019).

La principal fuente para la cronología del primer caso y las cuarentenas es Wikipedia, que se basa en fuentes hemerográficas. En la medida de lo posible esa información se contrastó con datos de prensa para confirmar la confiabilidad.

\section{CRONOLOGÍA BÁSICA}

La COVID-19 aparece en Wuhan en diciembre de 2019. Solo en la segunda semana de enero se reportan casos en otros países. El primero en Tailandia, el 13 de enero, y el 16 en Japón. El 20 de enero se reportan en Corea del Sur y Estados Unidos. En esa primera semana, el contagio abarca Asia Oriental y alcanza países desarrollados más lejanos; el patrón se repite la siguiente semana, cuando se reporta en Asia Suroriental (Taiwán, Hong Kong, Singapur, Vietnam, Malasia), Nepal y en países del primer mundo (Francia, Canadá, Alemania). En las dos semanas siguientes aparece en los demás países europeos, mediterraneos y escandinavos, y en Latinoamérica (en Brasil y México el 25 y el 28 de febrero). A Suramérica y el Caribe llega días más tarde, primero a Ecuador (febrero 29) y República Dominicana (marzo 1), y luego, entre el 3 y el 10 de marzo, a siete países suramericanos y a Europa central. Entre el 11 y el 19 de marzo aparece en islas del Caribe y América central. Después se detectará en África.

La primera oleada (13 al 23 de febrero) se concentró en países asiáticos que 1) sufrieron y aprendieron de epidemias respiratorias anteriores como el SARS; 2) por razones culturales, guardaban mayor 
distancia social (Sorokowska et al., 2017; Hall, 1966); y 3) eran de ingreso alto o medio. Por ello, a pesar de no haber recurrido a cuarentenas estrictas, Taiwán, Japón, Korea y Hong Kong controlaron la epidemia con medidas efectivas de cerco epimemiológico. Otros, como Nepal, Tailandia, Vietnam y Singapur, decretaron cuarentenas solo a finales de marzo o comienzos de abril, dos meses después de presentarse el primer caso. Estas no fueron muy largas y tuvieron diferentes duraciones, de 21 días en Vietnam a dos meses y medio en Nepal. Otra característica en varios de esos países y en China, fue la adopción de medidas de control de la población. En China se decretaron estrictas cuarentenas en el foco de la infección, lo que limitó el brote principalmente a la zona de Wuhan. La primera oleada también afectó a Estados Unidos, aunque por ser un país grande, los efectos tardarían en verse. Allí, las cuarentenas también empezaron tarde, entre el 19 y el 24 de marzo (dos meses después del primer caso) y solo en ocho estados; en cuatro de ellos terminaron después de uno o dos meses. Aunque hay similitud en la poca importancia que en conjunto se dio a las cuarentenas, en este país la diferencia fue que no se hizo cerco epidemiológico, ni tamizaje masivo, y la presidencia abrazó un discurso negacionista acerca de la pandemia.

La segunda oleada (febrero 24 a 2 de marzo) se concentró en Europa. Allí llegó a 19 países de Europa Occidental y a Rusia. La ausencia de fronteras en la zona Euro facilitó la difusión del virus, que también llegó a los países escandinavos. Igual que en la primera oleada, eran países de ingreso alto con un elevado índice de desarrollo humano, y con algunos de los sistemas sanitarios más sólidos del mundo. No obstante, en general no estaban preparados para una epidemia de tal magnitud y no tenían la experiencia de epidemias como el SARS o el ébola. La gran movilidad dentro de la zona Euro, la importancia del turismo y la poca distancia social en los países mediterráneos aceleraron la difusión del virus, y la inexperiencia hizo perder un tiempo valioso para la acción. El primer país en reaccionar fue Italia, que el 9 de marzo impuso una cuarentena que duró más de dos meses. Los demás países que adoptaron cuarentenas las impusieron entre el 14 y el 18 de marzo. Un poco después, Rusia y Alemania establecieron cuarentenas más cortas y medidas más relajadas. Otros países -como Canadá, Finlandia, Reino Unido, Suecia y Brasil- no impusieron cuarentenas. Suecia y Reino Unido defendieron un modelo de inmunidad de grupo, cuya lógica era dejar que la población se contagiara libremente, como ocurre con la gripe estacional. Cuando una alta proporción de la población se infecta y se vuelve inmune al 
virus, disminuye la probabilidad de contagio de la población restante. Este modelo pretendía minimizar el impacto en la economía, con un sólido sistema de salud que pudiera tratar a los enfermos.

Esta oleada también llegó a dos países grandes de América Latina (México y Brasil). Por su tamaño, los efectos tardarían en notarse, y junto a las políticas negacionistas de sus gobernantes, se agravó el problema. En ese momento era más bien invisible. Ecuador y República Dominicana son países mucho más pequeños, con un nivel de ingreso menor y flujos importantes de viajeros por migración y turismo. Además, Ecuador estaba sumido en una crisis política, agravada por la caída de los precios del petróleo, su principal producto de exportación. Por su tamaño, la crisis estallaría pronto en estos países, catastróficamente en Guayaquil. Un ejemplo temprano para otros países y las demás ciudades de Ecuador. E1 16 de marzo Ecuador impuso una cuarentena temprana por mes y medio, dos semanas después del primer caso. República Dominicana no impuso cuarentena. Aunque la COVID-19 llegó a Marruecos el 2 de marzo, seguir su trayectoria en África es más complicado. Debido a los niveles de pobreza y a la guerra, en varios países las cifras no reflejan la magnitud real del problema. Estos países ameritan un estudio propio.

La tercera oleada (del 3 al 10 de marzo) se concentra en Suramérica y Europa del Este, y llega a Panamá, Costa Rica y Turquía. Son países de ingreso medio y, en América Latina, con poca distancia social (Sorokowska et al., 2017). Ante la noticias del colapso en Italia, Ecuador y República Dominicana, otros países -como Perú, Argentina, Paraguay, Bolivia, Costa Rica y Colombia- inician cuarentenas tempranas, estrictas y prolongadas entre el 16 y el 25 de marzo (menos de un mes después del primer caso). Chile, que se encontraba en una crisis política, optó por medidas más laxas. Dos características de los países latinoamericanos son la desigualdad en la distribución del ingreso y la informalidad en el empleo. A pesar de las altas tasas de crecimiento que venía presentando Perú (4\% en 2018 y 2,2\% en 2019), las caracterísiticas mencionadas crearon dificultades para cumplir la cuarentena. Una gastronomía basada en comida fresca, unida a una pobreza que implicaba bajo número de refrigeradores, provocaba una alta y constante afluencia a los mercados públicos que se convirtieron en focos de infección (Jaramillo y Nopo, 2020).

La cuarta oleada se concentra en países del Caribe y Centroamérica. Algunos impusieron cuarentenas casi inmediatas. De allí en adelante, los países que tuvieron su primer contagio en el segundo trimestre caen por fuera del marco de análisis. 


\section{IMPACTO ECONÓMICO DE LA COVID-19}

Como ya se indicó, se establecieron cinco grupos de países para los que se construyeron series con los datos reportados por los gobiernos y las agencias de estadística nacionales ${ }^{1}$. La hipótesis central es que los elementos distintivos de cada grupo permiten verificar algunas de las conjeturas sobre el grado de afectación económica provocado por las políticas para contener la pandemia. Las variables utilizadas como aproximación del nivel de afectación económica son: la caída del PIB en el segundo trimestre de 2020, cuando la mayoría de los países de la muestra había implementado las medidas de contención, y la pérdida de empleos, medida como la caída de la tasa de ocupación.

\section{Gráfica 1}

Correlación entre contracción del PIB en 2020-II y muertes por COVID-19 (Por millón de habitantes)

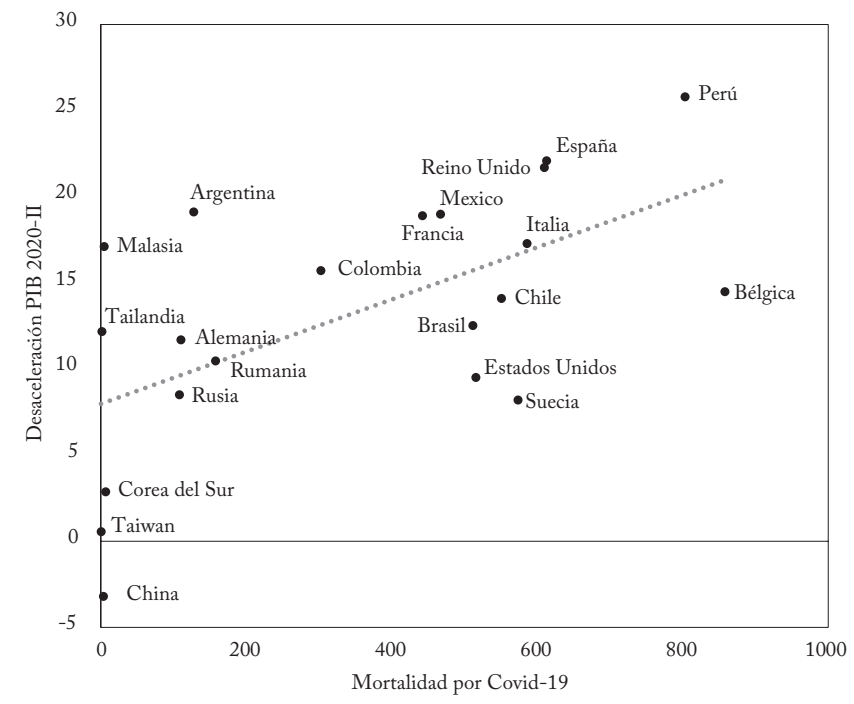

Fuente: agencias de estadística y bancos centrales nacionales; cálculos propios.

Cuando se observa la muestra total de países se identifican algunos hechos de contexto que arrojan luces sobre los diversos modos en que los países fueron afectados por las medidas de contención. Los costos se pueden dividir en costos en mortalidad y costos en creci-

${ }^{1}$ Los 29 países para los que se consiguió la información fueron: México, Rusia, Colombia, Irán, Iraq, Bolivia, Ecuador, Argentina, Malasia, Perú, Tailandia, Australia, Bélgica, Chile, Rumania, Suecia, Taiwán, Canadá, Alemania, Corea del Sur, España, Francia, Reino Unido, Italia, Bangladesh, Brasil, China, India, Estados Unidos. 
miento. La gráfica 1 muestra los costos en mortalidad (por millón de habitantes en el eje $\mathrm{x}$ ); $\mathrm{y}$ los costos económicos (contracción del PIB en el segundo trimestre de 2020 en el eje y). Si hubiese un trade-off entre las dos variables, como a veces se afirma, su correlación sería negativa. No obstante, la correlación es positiva (0,35). Países como Perú, España, Reino Unido y Bélgica han pagado un alto costo en vidas y en crecimiento, mientras que China, Taiwán y Corea del Sur tuvieron pocas muertes y su economía se vio afectada en menor medida. Parece que el buen o mal manejo de los aspectos sanitarios se tradujo en mejores o peores resultados económicos.

Esto se confirma cuando se observan las pérdidas de empleo medidas por la variación de las tasas de ocupación (gráfica 2). La magnitud de la pérdida de empleo es el porcentaje de reducción en la tasa de ocupación entre el cierre de 2019, y el final del primer semestre de 2020, (una reducción de la ocupación se representa por un número positivo que indica su magnitud). Pero a diferencia de la gráfica 1, aquí no parece haber correlación de ningún signo sino una gran variabilidad de los datos. De modo que no existe un trade-off. Los países con mayor caída de la ocupación son los de alta informalidad, como Colombia, Chile y Perú ${ }^{2}$. Lo que pudo haber ocurrido es que la pandemia hizo más visible el desempleo disfrazado (trabajo informal en sectores de baja productividad; Robinson, 1936). Es posible entonces que los datos de estos tres países sobreestimen el efecto de la pandemia sobre el empleo. Lo que sí es claro es que en ellos un gran número de personas carecían de mecanismos institucionales de protección, como las cesantías y los seguros de desempleo.

Otro grupo de países de la gráfica 2 es el de Canadá, Brasil y Estados Unidos, en el que al menos Estados Unidos y Brasil tuvieron gobiernos negacionistas, y se vieron forzados a cerrar la economía ante el colapso de sus sistemas de salud (en algunos estados), con significativas pérdidas de empleo y un elevado nivel de muertes por millón de habitantes. Canadá es un caso particular, pues el contagio inicial ocurrió en sus dos provincias más pobladas (Ontario y Quebec), pero a mediados de marzo se declaró el estado de emergencia en todos los gobiernos intermedios. Por ello hubo diversos grados de cierre de escuelas y guarderías, prohibiciones de reuniones, cierres de negocios no esenciales, restricciones de entrada y autoaislamiento obligatorio para los viajeros. Estas medidas afectaron la economía canadiense, y

${ }^{2}$ No se obtuvieron buenos datos de la tasa de ocupación de Bolivia, pero la tasa de desempleo pasó del 4,8\% a finales de 2019 al 8,6\%. en el segundo trimestre de 2020. 
ejercieron una presión para aumentar los despidos, que el gobierno intentó atenuar con un subsidio de salarios de emergencia (Government of Canada, 2020) y un estricto control epidemiológico. A pesar de ello, en el mes de mayo la tasa de desempleo llegó a su nivel más alto desde 1976: 13,5\%.

\section{Gráfica 2}

Correlación entre caída de la tasa de ocupación y muertes por COVID-19 (Por millón de habitantes)

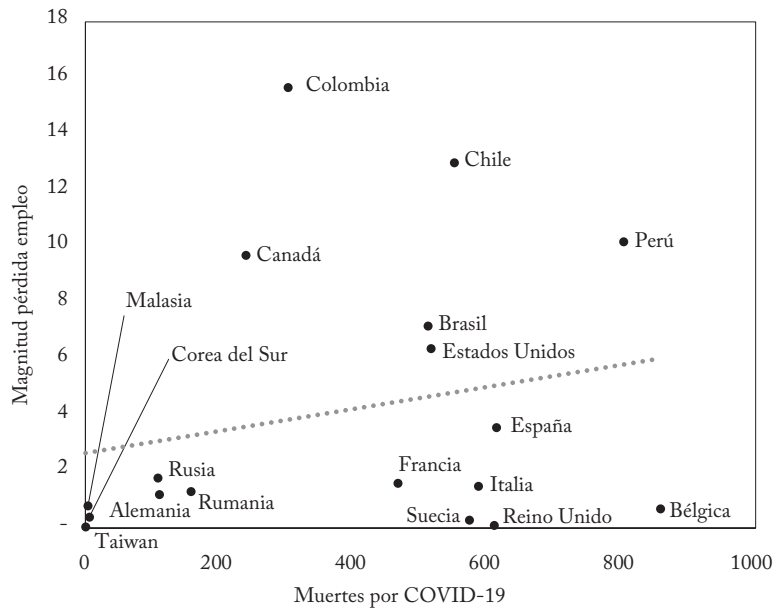

Fuente: agencias de estadística y bancos centrales nacionales; cálculos propios.

E1 tercer grupo identificado en la gráfica 2 es el de países con una pérdida de empleos menor, pero niveles heterogéneos de muertes. A la izquierda se observan los países asiáticos que, como ya se explicó, tenían experiencia en el manejo de epidemias similares, adoptaron políticas de tamizaje de la población y cercos epidemiológicos, y tenían prácticas de distanciamiento social por razones culturales (Hall, 1963).

Junto a estos países están Alemania, Rusia y Rumania, con una tasa de mortalidad un poco superior. Rusia y Alemania establecieron cuarentenas más cortas y medidas más relajadas. En Rusia las medidas de prevención temprana incluyeron un mayor control de la frontera con China y pruebas exhaustivas; después se tomaron medidas adicionales: cancelación de eventos, cierre de escuelas, teatros y museos, cierre de las fronteras y declaración de un período no laborable que se prolongó hasta el 11 de mayo. Alemania ha logrado una tasa de mortalidad menor debido al mayor número de pruebas realizadas, al mayor número de camas de cuidados intensivos disponibles con soporte respiratorio y a la mayor proporción de casos positivos entre 
los más jóvenes. Por estas razones y por el hecho de que en abril cerca de 500 mil empresas habían asignado a sus trabajadores un plan de trabajo de corta duración subsidiado por el gobierno, conocido como Kurzarbeit, se atenuó la presión sobre la pérdida de empleos. Por su parte, Rumania tomó medidas anticipadas para preparar su sistema de salud antes de la primera muerte, con cierres parciales posteriores que fueron levantados con relativa rapidez.

A la derecha y en la parte baja de la gráfica 2 se encuentran países europeos como Italia y España, donde colapsaron los sistemas de salud mientras las cuarentenas obligaban a parar la actividad económica. No obstante, estos países tenían espacio fiscal para enfrentar la pandemia y sistemas laborales que atenuaron en parte la presión para destruir empleos.

En la gráfica 3 se muestra la correlación entre la contracción del PIB en el segundo trimestre y el nivel de deuda pública anterior a la pandemia (2019) como porcentaje del PIB, como proxy del espacio fiscal de los países al inicio de la pandemia.

Gráfica 3

Correlación entre contracción del PIB en 2020-II y nivel de deuda en 2019

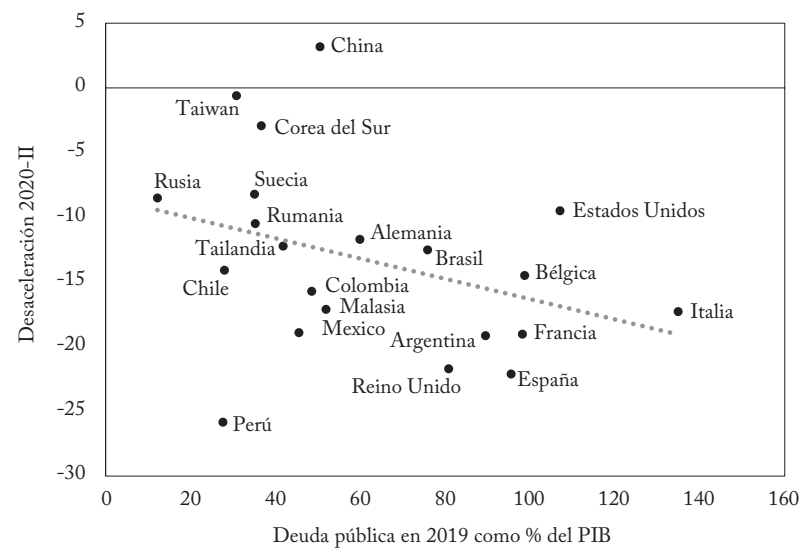

Fuente: agencias de estadística y bancos centrales nacionales; cálculos propios.

La posición fiscal, en términos de deuda pública ${ }^{3}$, muestra una correlación significativa con la contracción económica ocasionada por

3 Se tomó la deuda pública sobre el PIB como proxy del espacio fiscal porque es menos cíclica que el déficit fiscal anual, que depende de los ciclos políticos. No obstante, esa medida tiene la debilidad de que los niveles óptimos de deuda pueden diferir entre países; aún así se puede interpretar como el nivel de espacio fiscal. 
las medidas para enfrentar la pandemia. Al parecer, los países que tenían mayor espacio fiscal pudieron ejecutar programas para evitar la destrucción de empleos y mantener algunos niveles de consumo final. Perú es un caso atípico, porque arrancó con un nivel muy bajo de endeudamiento y tuvo la mayor contracción del PIB.

Dada el heterogéneo comportamiento de las cifras de empleo, actividad económica y muertes por COVID-19, se estimaron dos familias simples de modelos: una para explicar las diferencias entre la contracción económica del segundo trimestre, y otra para explicar la variabilidad de la pérdida de empleos. Como proxy de la capacidad instalada y de la preparación del sistema de salud se usó el número de camas por mil habitantes antes de la pandemia. Además, se utilizó el nivel de presión fiscal (deuda sobre PIB), como inverso del margen de maniobra de los países para hacer frente a la caída de la actividad económica; también se incorporaron el PIB per cápita de paridad de poder adquisitivo, como aproximación del nivel de riqueza, y el nivel de apertura ${ }^{4}$ como porcentaje del PIB, como medida de la dependencia externa de los países. Con estas variables y con las limitaciones naturales de una muestra pequeña se estimaron los modelos siguientes.

Cuadro 2

Modelos de impacto económico

Caída anual del PIB, 2020-II

\begin{tabular}{|c|c|c|c|c|c|c|}
\hline & Modelo 1 & Modelo 2 & Modelo 3 & Modelo 4 & Modelo 5 & Modelo 6 \\
\hline \multirow{2}{*}{$\begin{array}{l}\text { log(Muertes por } \\
\text { COVID-19/millón } \\
\text { de habitantes) }\end{array}$} & $2,60^{* * * *}$ & $1,47^{* * *}$ & $1,45^{* *}$ & $1,65^{* * * * *}$ & $1,79^{* * * *}$ & $1,66^{* * * *}$ \\
\hline & $(0,34)$ & $(0,57)$ & $(0,57)$ & $(0,37)$ & $(0,42)$ & $(0,52)$ \\
\hline \multirow{2}{*}{$\begin{array}{l}\log (\mathrm{Camas} / 1.000 \\
\text { hab. pre-CO- } \\
\text { VID-19) }\end{array}$} & 0,10 & $-3,69^{* * * *}$ & $-4,10^{* *}$ & $-4,46^{* * * *}$ & $-3,99 * * *$ & $-3,43^{*}$ \\
\hline & $(1,13)$ & $(1,23)$ & $(1,79)$ & $(1,46)$ & $(1,41)$ & $(1,80)$ \\
\hline \multirow{2}{*}{$\begin{array}{l}\log (\text { Deuda pública } \% \\
\text { PIB, 2019) }\end{array}$} & & $2,78^{* * *}$ & 1,98 & & & 2,70 \\
\hline & & $(1,01)$ & $(2,34)$ & & & $(2,47)$ \\
\hline \multirow{2}{*}{$\begin{array}{l}\text { log(PIB per cápita } \\
\text { PPP, 2019) }\end{array}$} & & & 0,37 & & $-0,70$ & $-1,97$ \\
\hline & & & $(0,94)$ & & $(1,07)$ & $(1,50)$ \\
\hline \multirow{2}{*}{$\begin{array}{l}\log (\text { Nivel de apertura } \\
\% \text { PIB, 2019) }\end{array}$} & & & & $2,73^{* * * *}$ & $4,16^{*}$ & $4,72^{*}$ \\
\hline & & & & $(0,68)$ & $(2,39)$ & $(2,50)$ \\
\hline Observaciones & 21 & 21 & 21 & 21 & 21 & 21 \\
\hline $\mathrm{R}^{2}$ & 0,84 & 0,89 & 0,89 & 0,90 & 0,90 & 0,91 \\
\hline $\mathrm{R}^{2}$ ajustado & 0,83 & 0,87 & 0,87 & 0,89 & 0,88 & 0,88 \\
\hline $\begin{array}{l}\text { Error residual } \\
\text { estándar }\end{array}$ & 6,26 & 5,31 & 5,44 & 5,08 & 5,18 & 5,11 \\
\hline Estadístico F & $50,85^{\text {**** }}$ & $49,98^{* * * *}$ & $35,74^{* * * *}$ & $55,16^{* * * *}$ & $39,94^{* * * *}$ & $33,13^{* * * *}$ \\
\hline
\end{tabular}

***: significativo al $1 \%$; **: significativo al $5 \%$; *: significativo al $10 \%$.

Fuente: agencias de estadística, bancos centrales nacionales y Our World in Data; cálculos propios.

${ }^{4}$ Exportaciones más importaciones. 
A pesar de disponer de un corte transversal y de un pequeño número de países con datos completos, los modelos indican ciertos fenómenos con una buena bondad de ajuste 5 . Primero, cuanto peor se ha manejado la pandemia, o cuantas más muertes se han presentado, mayor ha sido la pérdida de producción y de empleos en los países de la muestra. Segundo, con mayores niveles de significancia estadística, la capacidad instalada pre-pandemia, medida por el número de camas de hospital por cada mil habitantes, tiene una correlación significativa con un menor impacto en la producción y en la pérdida global de empleos. Lo que muestra esta cifra es que los países con sistemas de salud más sólidos pudieron darse el lujo de no realizar cuarentenas tan estrictas, tuvieron mayor velocidad de reacción, y lograron menor perjuicio económico que los países que tenían menor capacidad instalada.

Cuadro 3

Modelos de impacto económico

Caída de la tasa de ocupación, 2020-II

\begin{tabular}{|c|c|c|c|c|c|c|}
\hline & Modelo 1 & Modelo 2 & Modelo 3 & Modelo 4 & Modelo 5 & Modelo 6 \\
\hline \multirow{2}{*}{$\begin{array}{l}\text { log(Muertes por } \\
\text { COVID }-19 / \text { millón } \\
\text { de habitantes) }\end{array}$} & $2,60^{* * * *}$ & $1,47^{* *}$ & $1,45^{* *}$ & $1,65^{* * * *}$ & $1,79^{* * * *}$ & $1,66^{* * * *}$ \\
\hline & $(0,34)$ & $(0,57)$ & $(0,57)$ & $(0,37)$ & $(0,42)$ & $(0,52)$ \\
\hline $\begin{array}{l}\log (\text { Camas/1.000 hab. } \\
\text { pre-COVID-19) }\end{array}$ & $\begin{array}{c}0,10 \\
(1,13)\end{array}$ & $\begin{array}{c}-3,69^{* * * *} \\
(1,23)\end{array}$ & $\begin{array}{l}-4,10^{* *} \\
(1,79)\end{array}$ & $\begin{array}{c}-4,46^{* * * *} \\
(1,46)\end{array}$ & $\begin{array}{c}-3,99^{* * * *} \\
(1,41)\end{array}$ & $\begin{array}{l}-3,43^{*} \\
(1,80)\end{array}$ \\
\hline \multirow{2}{*}{$\begin{array}{l}\text { log(Deuda pública \% } \\
\text { PIB, 2019) }\end{array}$} & & $2,78^{* * * *}$ & 1,98 & & & 2,70 \\
\hline & & $(1,01)$ & $(2,34)$ & & & $(2,47)$ \\
\hline log(PIB per cápita & & & 0,37 & & $-0,70$ & $-1,97$ \\
\hline PPP, 2019) & & & $(0,94)$ & & $(1,07)$ & $(1,50)$ \\
\hline $\log$ (Nivel de apertura & & & & $2,73^{* * * * *}$ & $4,16^{*}$ & $4,72^{*}$ \\
\hline$\%$ PIB, 2019) & & & & $(0,68)$ & $(2,39)$ & $(2,50)$ \\
\hline Observaciones & 21 & 21 & 21 & 21 & 21 & 21 \\
\hline $\mathrm{R}^{2}$ & 0,84 & 0,89 & 0,89 & 0,90 & 0,90 & 0,91 \\
\hline $\mathrm{R}^{2}$ ajustado & 0,83 & 0,87 & 0,87 & 0,89 & 0,88 & 0,88 \\
\hline $\begin{array}{l}\text { Error residual es- } \\
\text { tándar }\end{array}$ & 6,26 & 5,31 & 5,44 & 5,08 & 5,18 & 5,11 \\
\hline Estadístico F & $50,85^{\text {***** }}$ & $49,98^{* * * *}$ & $35,74^{* * * *}$ & $55,16^{* * * *}$ & $39,94^{\text {***k }}$ & $33,13^{* * * *}$ \\
\hline
\end{tabular}

***: significativo al 1\%; ${ }^{* *}$ : significativo al $5 \%$; *: significativo al $10 \%$.

Fuente: agencias de estadística, bancos centrales nacionales y Our World in Data; cálculos propios.

Otro hecho interesante es la relación entre el nivel de apertura de las economías en 2019 y el mayor daño económico, tanto en producción como en empleo, lo que es comprensible pues las medidas adoptadas

5 Todos los errores estándar se corrigieron con errores robustos a la heteroscedasticidad (White, 1980). 
afectaron el comercio internacional. Por último, la relación entre el nivel de holgura fiscal y el daño económico no es concluyente.

La contracción de la actividad económica y la destrucción de empleos se produjeron principalmente por las medidas de contención de la pandemia. Al mismo tiempo, la mayor capacidad instalada en salud de algunos países en el periodo anterior a la pandemia funcionó como un amortiguador del impacto económico de ésta. Un ejercicio adicional para complementar el resultado anterior es comprender la varianza en las muertes por COVID-19 de la muestra, las cuales mostraron tener relación con el grado de impacto en la actividad económica y en la destrucción empleos.

Estos modelos muestran que, en los países de la muestra, el mayor número de muertes por COVID-19 tuvo relación con la preparación del sistema de salud medida por la capacidad instalada, con la proporción de población mayor de 65 años -la de mayor riesgo de muerte-, y con una menor densidad de población, por la dificultad para tomar y hacer cumplir las medidas de confinamiento. La falta de significancia del PIB per cápita muestra que la varianza de las muertes por COVID-19 de la muestra poco tiene que ver con la riqueza al inicio de la pandemia.

Cuadro 4

Modelo de muertes por COVID-19

(Por millón de habitantes)

\begin{tabular}{lcc}
\hline & Modelo 1 & Modelo 2 \\
\hline $\log ($ Camas/1.000 hab. pre-COVID-19) & $-1,73^{* * *}$ & $-1,64^{* *}$ \\
& $(0,75)$ & $(0,78)$ \\
$\log (\%$ Población > 65 años $)$ & $4,44^{* * * *}$ & $3,97^{* * *}$ \\
& $(0,72)$ & $(1,41)$ \\
Densidad poblacional (por km $\left.{ }^{2}\right)$ & $-1,07^{* *}$ & $-1,10^{* *}$ \\
& $(0,42)$ & $(0,45)$ \\
$\log ($ PIB per cápita PPP, 2019) & & 0,12 \\
Observaciones & 21 & $(0,33)$ \\
$\mathrm{R}^{2}$ & 0,88 & 21 \\
$\mathrm{R}^{2}$ ajustado & 0,86 & 0,88 \\
Error residual estándar & $1,97(\mathrm{df}=18)$ & 0,85 \\
Estadístico F & $43,93^{* * * *}(\mathrm{df}=3 ; 18)$ & $31,28^{* * * *}(\mathrm{df}=4 ; 17)$ \\
\hline
\end{tabular}

***: significativo al 1\%; **: significativo al $5 \%$; * significativo al $10 \%$.

Fuente: agencias de estadística, bancos centrales nacionales y Our World in Data; cálculos propios.

Para consolidar el análisis se hace una síntesis de los grupos por tamaño poblacional, nivel de ingreso y dependencia del petróleo. 
Cuadro 5

Impactos económicos por grupo de países

\begin{tabular}{|c|c|c|c|c|c|c|c|c|}
\hline País & Grupo & $\begin{array}{l}\text { Fecha } \\
\text { primer } \\
\text { contagio }\end{array}$ & 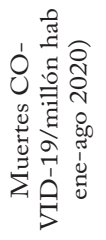 & 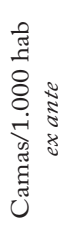 & 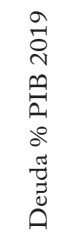 & 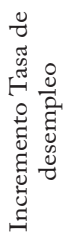 & 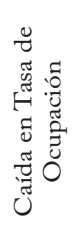 & 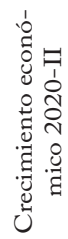 \\
\hline México & \multirow{7}{*}{$\begin{array}{l}\text { Exporta- } \\
\text { dores de } \\
\text { hidrocar- } \\
\text { buros }\end{array}$} & $28 / 02 / 2020$ & 442,3 & 1,5 & 45,5 & ND & ND & $-18,9$ \\
\hline Rusia & & $31 / 01 / 2020$ & 107,9 & 8,2 & 12,2 & 1,6 & $-1,8$ & $-8,5$ \\
\hline Colombia & & 6/03/2020 & 302,1 & 1,5 & 48,4 & 9,4 & $-15,7$ & $-15,7$ \\
\hline Irán & & $19 / 02 / 2020$ & 235,8 & 1,5 & 44,2 & ND & ND & ND \\
\hline Iraq & & $24 / 02 / 2020$ & 148,0 & 1,4 & 48,4 & ND & ND & ND \\
\hline Bolivia & & $10 / 03 / 2020$ & 353,2 & 1,1 & 57,7 & ND & $-0,5$ & ND \\
\hline Ecuador & & $29 / 02 / 2020$ & 344,8 & 1,5 & 49,4 & ND & ND & ND \\
\hline Argentina & \multirow{4}{*}{$\begin{array}{l}\text { Ingreso } \\
\text { medio alto, } \\
\text { tamaño } \\
\text { mediano }\end{array}$} & $3 / 03 / 2020$ & 127,2 & 5 & 89,4 & 5,6 & ND & $-19,1$ \\
\hline Malasia & & $25 / 01 / 2020$ & 3,9 & 1,9 & 51,8 & 1,6 & $-0,8$ & $-17,1$ \\
\hline Perú & & $6 / 03 / 2020$ & 803,1 & 1,6 & 27,5 & 10,2 & $-10,2$ & $-25,8$ \\
\hline Tailandia & & $13 / 01 / 2020$ & 0,8 & 2,1 & 41,8 & $\mathrm{ND}$ & ND & $-12,2$ \\
\hline Australia & \multirow{6}{*}{$\begin{array}{l}\text { Ingreso } \\
\text { alto, } \\
\text { tamaño } \\
\text { pequeño }\end{array}$} & $25 / 01 / 2020$ & 16,5 & 3,8 & 45,1 & 2,3 & ND & ND \\
\hline Bélgica & & $4 / 02 / 2020$ & 858,0 & 6,2 & 98,6 & 0,3 & $-0,7$ & $-14,5$ \\
\hline Chile & & $3 / 03 / 2020$ & 550,0 & 2,2 & 27,9 & 5,1 & $-13,0$ & $-14,1$ \\
\hline Rumania & & $26 / 02 / 2020$ & 157,5 & 6,3 & 35,2 & 1,2 & $-1,3$ & $-10,5$ \\
\hline Suecia & & $31 / 01 / 2020$ & 573,0 & 2,6 & 35,1 & 3,8 & $-0,3$ & $-8,2$ \\
\hline Taiwán & & $21 / 01 / 2020$ & 0,3 & 5,7 & 30,8 & 0,3 & $-0,1$ & $-0,6$ \\
\hline Canadá & \multirow{7}{*}{$\begin{array}{l}\text { Ingreso } \\
\text { alto, } \\
\text { tamaño } \\
\text { mediano }\end{array}$} & $25 / 01 / 2020$ & 239,3 & 2,7 & 89,7 & 6,7 & $-9,7$ & ND \\
\hline Alemania & & $27 / 01 / 2020$ & 110,2 & 8,3 & 59,8 & 0,9 & $-1,2$ & $-11,7$ \\
\hline Corea del Sur & & $20 / 01 / 2020$ & 6,0 & 11,5 & 36,6 & 0,6 & $-0,4$ & $-2,9$ \\
\hline España & & $31 / 01 / 2020$ & 612,7 & 3 & 95,5 & 1,5 & $-3,6$ & $-22,1$ \\
\hline Francia & & $24 / 01 / 2020$ & 466,2 & 6,5 & 98,1 & $(1,0)$ & $-1,6$ & $-19,0$ \\
\hline Reino Unido & & $31 / 01 / 2020$ & 609,4 & 2,8 & 80,7 & 0,1 & $-0,1$ & $-21,7$ \\
\hline Italia & & $30 / 01 / 2020$ & 585,5 & 3,4 & 134,8 & $(0,7)$ & $-1,5$ & $-17,3$ \\
\hline Brasil & \multirow{4}{*}{$\begin{array}{l}\text { Muy } \\
\text { grandes }\end{array}$} & $25 / 02 / 2020$ & 510,6 & 2,2 & 75,79 & 2,3 & $-7,2$ & $-12,5$ \\
\hline China & & $1 / 12 / 2020$ & 3,3 & 4,2 & 50,5 & 0,5 & ND & 3,2 \\
\hline India & & $30 / 01 / 2020$ & 37,5 & 0,7 & 69,62 & 3,4 & ND & ND \\
\hline Estados Unidos & & $20 / 01 / 2020$ & 515,1 & 2,9 & 106,9 & 7,6 & $-6,4$ & $-9,5$ \\
\hline
\end{tabular}

***: significativo al 1\%; **: significativo al 5\%; *: significativo al $10 \%$.

Fuente: agencias de estadística, bancos centrales nacionales y Our World in Data; cálculos propios.

Para los países de ingreso alto y tamaño mediano, se puede decir que los efectos, en salud y en la economía, dependieron de la preparación. Corea estaba preparada; a los demás países los cogió de sorpresa. Tenían muy buenos sistemas de salud y buen nivel de ingreso, pero les fue mal, incluso cuando dieron prioridad a la economía. No obstante, la destrucción de empleos solo fue grave en Canadá, que como se explicó antes tuvo otro enfoque para enfrentar la pandemia. 
Como se observa en el cuadro 5, la contracción económica en España (22,1\%), Reino Unido (21,7\%), Francia (19\%) e Italia (17,3\%) contrasta con la menor caída de Alemania (11,7\%), que recurrió al testeo masivo y cuyo sistema de salud tenía buena capacidad: 8,3 camas frente a 3,7 en los demás países europeos y a 11,5 en Corea.

En los países de ingreso alto y tamaño pequeño todo dependió de la capacidad y voluntad para hacer cuarentena estricta o controlar a la población. Solo Chile registró una fuerte destrucción de empleos, una caída de la tasa de ocupación del 13\%, debido quizá a los diferenciales de informalidad. Empero, la mayoría de los países ${ }^{6}$ tuvo caídas significativas de la actividad económica, incluso Suecia que siguió el modelo de inmunidad de grupo y tuvo muchas muertes en ancianatos. El país que hace la diferencia en este grupo es Taiwán, tanto en la mortalidad como en los efectos económicos, lo que respalda la hipótesis de que la preparación ex ante del sistema de salud, no solo por el número de camas, sino por la logística para enfrentar pandemias recurrentes, atenuó los perjuicios económicos de la pandemia.

En los países de ingreso medio alto y de tamaño mediano existía mayor capacidad de maniobra que en los países petroleros. No obstante, Argentina venía con una recesión de casi dos años seguidos y Perú había experimentado una crisis política, con conflictos entre el poder legislativo y el ejecutivo, sin olvidar el alto nivel de informalidad. En estos dos países el PIB se contrajo -un 19,1\% en Argentina y un 25,8\% en Perú- y aumentó la tasa de desempleo -casi 5 puntos porcentuales en Argentina y el 10,2\% en Perú-debido principalmente al alto nivel de informalidad y a la situación de pobreza mencionada en la sección anterior. Los países asiáticos de este grupo (Malasia y Tailandia) tuvieron una menor contracción de la actividad económica (cuadro 5); Malasia solo registró un incremento del 1,6\% en la tasa de desempleo y una baja de la tasa de ocupación de 0,8 puntos porcentuales. La diferencia entre estos países del grupo de ingreso medio alto y de tamaño mediano obedece sobre todo a la preparación para enfrentar pandemias (Malasia y Tailandia), y a los niveles de informalidad. Mientras que Perú, Argentina y Tailandia tienen altos niveles de informalidad (60\%, 49\%, y 37\%, respectivamente), Malasia solo tiene un $10 \%$, lo cual se traduce en una menor destrucción de empleo durante la respuesta a la COVID-19.

Por su parte, los países muy grandes se comportan como una colección de países y la evolución fue muy lenta. No hay muchos datos

${ }^{6}$ Incluimos a Australia en este grupo porque pese a ser muy extensa, su población es pequeña y se agrupa en las ciudades costeras. 
de India, pero al parecer su tasa de mortalidad por millón no es muy alta y hay grandes disparidades entre los distintos estados. Como ya se mencionó, en China se han hecho cuarentenas estrictas en los focos de contagio, que han minimizando el impacto sobre la economía y el empleo. A los negacionistas, como Estados Unidos y Brasil, les fue mal tanto en lo económico como en lo sanitario: al inicio decidieron no cerrar, pero una vez se hizo ver la presión sobre los sistemas de salud, permitieron cuarentenas en los niveles subnacionales. El efecto diferencial entre Brasil y Estados Unidos puede deberse a los distintos grados de capacidad sanitaria instalada antes de la pandemia (baja en ambos países) y de informalidad laboral.

Gráfica 4

Evolución reciente de los precios del petróleo

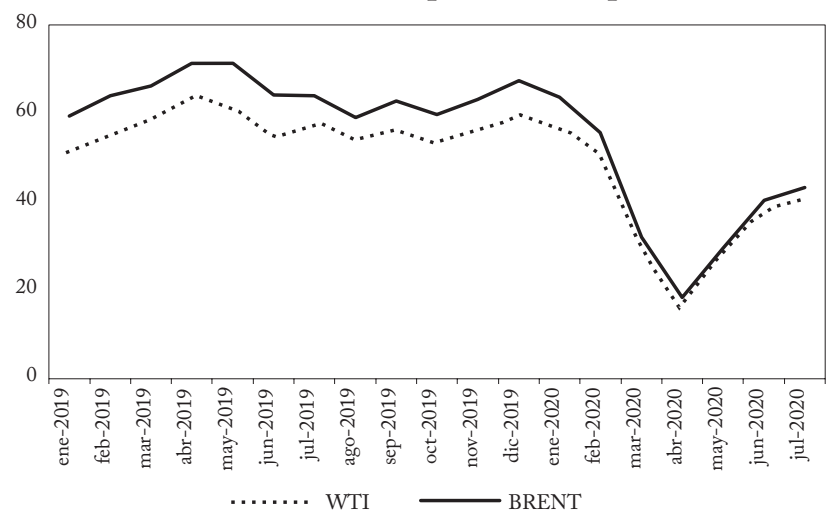

Fuente: EIA.

Finalmente, la tendencia a la baja de los precios del petróleo durante el primer semestre de 2020, producida por el desacuerdo entre $\mathrm{Ru}-$ sia y Arabia Saudita y luego por las medidas iniciales tomadas por muchos países para contener la pandemia, produjo un doble choque a los países exportadores de petróleo. E1 precio del barril WTI pasó de 57,2 dólares en enero a un mínimo de 16,5 dólares en abril, y el crudo de referencia BRENT siguió una trayectoria similar, de 63,6 a 18,4 dólares en el mismo periodo. Pese a que los precios del petróleo se han recuperado paulatinamente con la reapertura de muchos países una vez pasado el pico de la pandemia, tal caída de precios redujo el espacio fiscal que podía hacer posible asumir los retos de la pandemia, tanto en el frente del gasto público para evitar la quiebra masiva de empresas y el aumento de la pobreza, como el gasto de inversión para ampliar la capacidad instalada del sector de la salud. 
E1 crecimiento económico tuvo una fuerte reducción en México Colombia y Rusia $(-18,9 \%,-15,7 \%, y-8,5 \%$ respectivamente). México venía de una recesión importante después de haberse contraído un $0,5 \%$ en el último trimestre de 2019 , y un $-1,4 \%$ en el primer trimestre de 2020. Las diferencias de los efectos económicos en los países petroleros de la muestra ${ }^{7}$ obedecieron a los distintos niveles de espacio fiscal y de formalidad de la economía.

Los costos en empleo fueron más bajos en Rusia que en Colombia y en México, sobre todo por las medidas adoptadas: una respuesta temprana en Rusia y una intervención fiscal para amortiguar el colapso económico. Allí se tomaron medidas para proteger el empleo en las microempresas y las pequeñas y medianas empresas: diferir los pagos de impuestos (excepto el IVA) durante seis meses, reducir a la mitad los aportes a la seguridad social, diferir las contribuciones a la seguridad social, y una moratoria de seis meses del pago de multas, cobro de deudas y solicitudes de acreedores para decidir la quiebra de las empresas deudoras. La mortalidad es un poco más baja en Colombia, pero los costos económicos son mucho más altos, por el poco espacio fiscal para evitar el colapso y el alto nivel de informalidad de la economía. Ecuador y Bolivia (este último exportador de gas natural) tuvieron su primer contagio casi al mismo tiempo. En el segundo trimestre Ecuador experimentó una caída anual del PIB del $17 \%$, asociada a la crisis de sus exportaciones y a la informalidad. No hay cifras de Bolivia para hacer comparaciones.

\section{¿EL FIN DE LA GLOBALIZACIÓN?}

La unidad de análisis de la sección anterior es el Estado-nación, pero en las últimas décadas la palabra clave en el comercio internacional fue "globalización". Proceso que cobró fuerza en este siglo con una nueva división del trabajo entre países en las cadenas globales de valor. La producción de un bien se segmentó entre distintos países, lo que en México y América Central se refleja en el auge de las maquilas. Pero es un fenómeno que se extiende a Asia, África y Europa.

Hay dos tipos de cadenas globales de valor: cadenas orientadas al comprador y cadenas orientadas al productor. En las cadenas orientadas al comprador, vendedores al detal de gran tamaño como Wal-Mart y marcas como Nike, que no tienen o tienen pocas fábricas propias, determinan la cadena de producción por el mero volumen de sus compras; debido a su poder de mercado la producción se ubica en

7 Para los países petroleros como Ecuador, Bolivia, Irak e Irán no se encontró información disponible para el segudo trimestre de 2020. 
lugares con bajos costos. Estas cadenas son más usuales en productos simples: juguetes, bienes para el hogar y ropa. La innovación se da más en el diseño y el mercadeo. La cadena se compone de firmas independientes, muchas de ellas ubicadas en países poco desarrollados, cuya ventaja comparativa es en esencia el bajo nivel de salarios. Aunque se han popularizado en países del Tercer Mundo, la participación de estos países en las cadenas genera ventajas y desventajas en las economías locales.

A nivel agregado, la participación de empresas en las cadenas globales de valor aumenta la productividad y mejora el ingreso del país, como sucedió en Vietnam. Allí, el sector de productos electrónicos tuvo una gran expansión que llevó a producir el 40\% de los productos de telefonía de Samsung a nivel mundial. Además, las mejoras en conectividad impulsaron la importación y la exportación, determinantes del éxito en esta cadena. Con la participación de Bangladesh en una cadena global las exportaciones de ropa y zapatos pasaron de menos del 1\% del PIB en 1988 al 14\% del PIB en 2016. Si bien la pobreza extrema se redujo del $44 \%$ al $15 \%$ en 2016, las bajas tasas de salarios, uno de los factores del aumento de las exportaciones de Bangladesh, provocaron protestas que pusieron de manifiesto las malas condiciones laborales en muchas fábricas asociadas a la cadena, así como los perjuicios ambientales de un sector que consume dos veces más agua que la población de Daca, la capital. Las demandas de los trabajadores para que se mejoren las condiciones laborales y de los consumidores para que se limite el impacto ambiental han impulsado la adopción de medidas para atenuar estos problemas. En todo caso, la cadena impulsa el aumento de la productividad y de los ingresos gracias a las relaciones de largo plazo con las firmas involucradas y la hiperespecialización en tareas específicas. La incorporación de estos dos países a la cadena global de valor se debió a los tratados de libre comercio, a la normatividad favorable para la inversión extranjera, a los bajos salarios y a la cercanía geográfica con oferentes de dispositivos y componentes electrónicos de buena calidad como China, Japón, Corea del Sur y Tailandia (World Bank, 2020).

En las cadenas orientadas al productor, firmas manufactureras como Toyota, General Motors o IBM fragmentan la producción entre afiliados de la misma firma multinacional. Tienden a crearse en bienes intensivos en tecnología y capital, como carros y electrónica compleja. En estas áreas las innovaciones se presentan en materia de know-how.

Costa Rica es un ejemplo interesante. Este país dio un gran salto que llevó de una economía basada en actividades productivas 
tradicionales a una basada en la industria y servicios de mediana y alta tecnología. Mediante políticas de apertura económica y medidas para atraer inversión extranjera directa se logró esa transformación productiva, en la que incidió el ingreso de Intel a Costa Rica en 1998 (Monge, 2017) para desarrollar actividades tecnológicas sofisticadas, lo que atrajo a otras firmas multinacionales para realizar operaciones.

La posibilidad de atraer capital extranjero a un país depende de lo que las empresas buscan. Algunas buscan eficiencia y otras recursos; el atractivo del país para ofrecer estas opciones facilita su integración a la cadena. En Costa Rica, empresas como Intel buscaban eficiencia y requerían mano de obra con competencias tecnológicas de nivel medio y alto. En 2014, Intel trasladó operaciones de ensamblaje y prueba de procesadores a Asia, pero dejó las labores de ingeniería y diseño en Costa Rica, lo que elevó su participación en la cadena. No obstante, la participación en estas cadenas aumenta la sensibilidad a choques por alteraciones del comercio internacional. La pandemia afectó las operaciones de exportación e importación en Costa Rica y las empresas empezaron a explorar otras posibilidades de producción de bienes y uso de capacidades; por ejemplo, Intel colaboró con el Seguro Social para elaborar modelos predictivos del comportamiento de la COVID-19 usando la capacidad de sus ingenieros para manejar datos, y ha impulsado iniciativas de capacitación en el manejo de herramientas tecnológicas y producción de respiradores.

El gran impacto de la COVID-19 en los flujos comerciales de los participantes en las cadenas globales de valor si bien parece novedoso no es nuevo. La crisis de 2008, el terremoto de 2011 en Japón, etc., ya habían enviado señales de la fragilidad del comercio internacional ante ciertas contingencias, frente a las cuales la mejor estrategia a seguir no es necesariamente reubicar las operaciones. Las empresas multinacionales que participan en estas cadenas se adaptan identificando oportunidades no solo para mejorar la eficiencia sino también su resiliencia. Aunque no hay una trayectoria estándar, algunas industrias se han concentrado en adaptar su infraestructura, sus procesos y capacidades para producir bienes relacionados con la salud; otras han ajustado sus procesos para producir bienes menos dependientes de insumos provenientes del exterior; y algunas empresas han convertido sus operaciones de abastecimiento en redes de abastacimiento, lo que ha implicado una gran transformación digital para incorporar el e-commerce, soportado por la conectividad y la infraestructura tecnológica, pero basado en la posibilidad de hacer 
alianzas entre empresas para acelerar la reactivación económica y elevar la productividad.

En general, las empresas están replanteando sus operaciones por la posibilidad y los riesgos de futuras contingencias -otras pandemias, cambio climático, desastres naturales, etc.- que expondrán al mundo a una mayor frecuencia de situaciones caóticas, en las que que se exhacerben los sentimientos proteccionistas y los llamados a acortar las cadenas o promover la autosuficiencia (Zhenwei et al., 2020).

Parece que las cadenas globales de valor tienden a ser más regionales. El peligro de desabastecimiento de bienes importados puede llevar a que los países busquen garantizar una producción local mínima para reducir su dependencia del comercio internacional.

\section{CONCLUSIONES}

Aunque el PIB inicial y el bajo endeudamiento daban mayor flexibilidad para adoptar medidas de mitigación, el PIB antes de la pandemia no es un buen predictor de resultados favorables en el manejo de la crisis. En cambio, un factor que atenuó la crisis económica y sanitaria fue la existencia de un buen sistema de salud.

No se encontró evidencia de trade-off entre reducción de las muertes por COVID-19 y actividad económica. La relación entre contracción del PIB y tasa de mortalidad por COVID-19 es positiva, quizá porque los países que en el primer semestre del año manejaron peor la crisis de salud pública (más muertes por millón de habitantes) se vieron obligados a tomar medidas de contención ex post más duras, o porque el colapso de los sistemas de salud tuvo efectos negativos en las decisiones de los agentes, lo que provocó una mayor contracción de la actividad económica.

Aunque no se encontró un trade-offentre mortalidad y pérdida de empleo, los países con peores caídas en la tasa de ocupación tendieron a ser los que tenían un alto nivel de empleo informal.

La COVID-19 mostró que no se puede depender totalmente de la importación de alimentos, medicamentos y, ni siquiera, de productos o servicios. Esto puede llevar a un nuevo tipo de proteccionismo en el futuro. Se debe garantizar una producción local mínima que impida el desabastecimiento en épocas de crisis.

La mejor preparación para la pandemia habría sido la adopción anterior de medidas sociales para fortalecer el sistema de salud y formalizar el empleo. Una lección que la pandemia deja a América Latina es que la prioridad no debería ser el crecimiento per se, sino la 
inversión para mejorar la prestación de bienes y servicios públicos, y eliminar la informalidad laboral y empresarial.

\section{REFERENCIAS BIBLIOGRÁFICAS}

Bradley, D. (2020). Despite mid-year bump, EIA expects 2020 natural gas prices to average Just \$2.11. Natural Gas Intelligence, [https://www. naturalgasintel.com/despite-mid-year-bump-eia-expects-2020-naturalgas-prices-to-average-just-2-11/].

Chiaramonti, D y Maniatis, K. (2020). Security od supply, strategic storage and COVID-19; Which lessons learnt for renewable and recycle carbon fuels, and their future role in decarbonizing transport? Applied Energy, 271(C), 1-20.

Government of Canada. (2020). Canada emergency wage subsidy, [[https:// www.canada.ca/en/revenue-agency/services/subsidy/emergency-wagesubsidy.html].

Jaramillo, M y Nopo, H. (2020). COVID-19 y shock externo: impactos económicos y opciones de política en el Perú. PNUD, Serie de Documentos de Politica Pública.

Hall, E. T. (1990). The hidden dimension. Nueva York, NY: Doubleday.

Mannucci, E., Nreu, B. et al. (2020). Factors associated with increased all-cause mortality during the COVID-19 pandemic in Italy. International Journal of Infectious Diseases, 98, 121-124.

Monge G., R. (2017). Ascendiendo en la cadena global de valor: el caso de Intel Costa Rica. Lima: OIT, Oficina Regional para América Latina y el Caribe.

Robinson, J. (1936). Disguised unemployment. Economic Journal, 182(46), 225-237.

Sorokowska, A. Sorokowski, P. et al. (2017). Preferred interpersonal distances: a global comparison. Journal of Cross-Cultural Psychology, 48(4), 577-592.

World Bank. (2020). Word Development Report 2020: Trading for development in the age of global value chains. Washington, DC: World Bank.

Zhenwei, C., Li, Y., et al. (2020). Foreign direct investment and global value chains in the wake of COVID-19: Lead firms of GVC, mensaje de un blog. World Bank Blogs, [https://blogs.worldbank.org/psd/foreigndirect-investment-and-global-value-chains-wake-covid-19-lead-firms-gvc]. 